\title{
Valeur taxonomique des caractères morphologiques et anatomiques du pore génital chez les Trématodes du genre Carmyerius (Gastrothylacidae)
}

\author{
par S. GRÉTILLAT \\ (Laboratoire National de Recherches vétérinalres de Dakar)
}

\begin{abstract}
RÉSUMÉ
La systématique des Gostrothylacidae, particulièrement celle des espèces du genre Cormyerius STILES et GOLBERGER, 1910, est plus ou moins confuse, tout au moins en ce qui concerne certaines espèces où les critères morphologiques sont fournis seulement par les dimensions des testicules, le diamère de l'acetabulum, etc..., caractères très fluctuants si l'on considère que dans ce groupe la contraction du matériel conservé peut influer consıdérablement sur l'aspect et les dimensions de certains organes.

Par contre. après avoir étudié la structure histologique du pore génital des diverses espèces du genre, il semble qu'il sait possible de classer ces dernières (tout au moins certaines d'entre elles) en prenant comme critères l'existence ou la non-existence, l'importance et les dimensions:

$1^{\circ}$ de l'atrium génital,

$2^{\circ}$ du sphincter atrial,

$3^{0}$ du sphincter papillaire.

Grâce à ces critères, il est possible de détermıner cerłainement onze espèces sur seize déjà décrites à l'heure actuelle.
\end{abstract}

Les trématodes du genre Carmyerius sont des helminthes de la famille des Gastrothylacidae STILES ET GOLDBERGER, 1910, du sous-ordre des Paramphistomordeo STYLES et GOLDBERGER, 1910, caractérisés par l'existence d'une poche centrale dont le volume peut afteindre le tiers de celui du corpr et dont l'ouverture intérieure débouche justr .n arrière de l'orifice buccal en position veni, ale. Les testicules qui se trouvent en position latéro-postérieure juste en avant et à côté du plafond de l'acetabulum, permettent de séparer les espèces du genre Car- myerius de celies du genre Fischoederius STILES et GOLDBERGER, 1910, où les testicules sont disposés l'un en avant de l'autre.

Dans le genre Gostrothylax POIRIER, 1883, les testicules sont en position latérale mais l'utérus croise en $X$ le canal déférent mâle dans la partie moyenne du corps alors que chez Cormyerius, ces deux canaux sont en position dorsale et cheminent parallèlement.

Le genre Carmyerius comprend actuellement 16 espèces, mais pour bon nombre d'entre elles, la diagnose est extrêmement difficile. En effet، les 
caractères pris par la majorıté des auteurs pour décrire les espèces, sont, bien sûr, les dimensions et l'emplacement de certains organes, tels que les testicules, l'ovaire, la glande de MEHLIS ou encore la position et le développement des glandes vitellogènes, la longueur de l'œsophage ainsi que le diamètre des ventouses orale et postérieure (acetabulum).

Or, suivant les spécimens et surtout suivant les milieux fixateurs dans lesquels ils ont été conservés, la position et les dimensions de ces différents éléments peuvent varier parmi les individus d'une même espèce, le tıssu aérolarre de soutien étant très lâche.

Nous avons donc pensé que peut-être, en ayant recours aux caractères histologiques et anatomiques d'un organe qussi différencié que le pore génital, Il pourraıt être possible d'établir une classification des différentes espèces de Carmyerius, en se basant sur des caractères moins fluctuants ef moins sujets à controverse que ceux utilisés jusqu'à ce jour.

Il est bien entendu que les autres éléments anatomiques sont toujours valables pour confirmer ou infirmer la diagnose dans certains cas douteux et particulièrement pour les deux ou trois espèces de Cormyerius chez lesquels le pore génital a pratiquement les mêmes structures histologique et anatomique.

R. Ph. DOLLFUS a tout récemment montré en 1963, combien est illusoire le critère des dimensions des aufs dans la diagnose des Carmyerius. Par contre cet auteur, en s'appuyant sur le diagramme que représente la poche ventrale en coupes transversales, a pu classer certains représentants de la famille des Gastrothylacidae. Malheureusement, tout comme pour la position des testicules, de l'ovaire et des autres organes, la figure géométrique représentant cette poche centrale, varie suivant le degré de constriction du matériel examiné et ne permet pas à notre avis, de classer d'une manière sûre, les différentes espèces de Carmyerius. DOLLFUS le fait d'ailleurs très justement remarquer : « Chez beaucoup d'espèces, la forme de la section transversale de la poche centrale, est inconstante ». La valeur taxonomique d'un tel critère est donc très relative et ne dolt être utilisée qu'accessoirement en tenant compte des caractères anatomiques et histologiques de certains organes qui sont par contre, beaucoup plus constants.

Après avoir examiné en détail, la morphologie et I'histologie du pore génıtal ou plutôt de l'aire génitale de 12 espèces du genre Carmyerıus, nous avons tenté de les classer d'après certains caractères tels que la présence ou l'absence d'un atrium génital, la présence ou l'absence d'un sphincter papıliaıre, la présence ou l'absence de papilles recouvrant les parois de l'atrium génltal ou de la zone papillare.

\section{ÉLÉMENTS ANATOMIQUES DU PORE GÉNITAL CHEZ LES CARMYERIUS}

Au centre de l'aire génitale, débouche un élément en forme de champignon, qui est l'arrıvée commune des conduits génitaux mâle et femelle. Cette partie est circonscrite par un épaississement musculaire qui peut dans certains cas prendre l'allure d'un sphincter plus ou moins puissant ef épais. Ce complexe sphincto-papillaire peut déboucher directement dans la partie dorso-antérieure de la poche centrale ou au contraire dans le fond d'un atrıum génital qui peut être vaste ou de dimensions réduites et qui s'ouvre lui-même dans la poche centrale. Nous appuyant sur ces différents caractères morphologiques, anatomıques et histologiques, nous avons pu établir une clé de détermınation permettant de faire une diagnose facile et à peu près certaine, des différentes espèces du genre Carmyerius.

Dans cette clé, ont été intentionnellement passées sous silence, les deux espèces $C$. bubalis (INNES, 1912) et C. cruciformis (LEIPER, 1910) en raison de l'absence totale de renseignements concernant la structure de leur pore génital, la seconde ayant d'ailleurs été décrite sur des spécimens immatures; le seul critère valable invoqué par l'auteur étant le diagramme en forme de croix que présente la poche centrale en coupe transversale.

Dans la classification que nous donnons cidessous, à part quelques petites variations portant sur des' détails mınel' I'ornementation et la musculature, la morphulogie et l'histologie du pore génital ef de la région qui l'entoure ont des caractères constants qui à notre avis, représentent de très bons critères. 


\section{CLÉ D'IDENTIFICATION DES ESPÈCES DU GENRE CARMYERIUS, BASÉE UNIQUEMENT SUR LA STRUCTURE DU PORE GÉNITAL}

1) Pore génital non orné de papilles..... 3

2) Pore génital orné de papilles.........

3) Pore génital débouchant dans le fond d'un atrium génital dont les parois possèdent une musculature propre. .

4) Pore génital débouchant dans le fond d'un atrium génital de petites dimensions et dont les parois ne présentent pas de musculature bien développée.

5) Pore génital sans atrium génital :

Cormyerius spatiosus (BRANDES, 1898), C. weimani (STILES et GOLDBERGER, 1910), C. gregorius (LOOSS, 1896) et $C$. minutus (FISCHOEDER, 1901).

6) Très grand atrium génital de 4 à $500 \mu$ de diamètre et de profondeur....... ...... C. synethes (FISCHOEDER, 1901)

7) Atrium génital de dimensions réduites.

8) Présence d'un puissant sphincter atrial bordant les marges de l'atrium génital..... C. graberi, (GRÉTILLAT, 1960)

9) Pas de sphincter atrial proprement dit. .. C. dollfusi (GOLVAN, CHABAUD et GRÉTILLAT, 1957)

10) Pore génital débouchant dans la poche ventrale C. mancupatus (FISCHOEDER, 1901)

11) Pore génital débouchant en dehors de la poche ventrale, sphincter atrial se confondant avec la musculature du pore génital .................. ... C. exoporus (MAPLESTONE, 1923)

12) Pore génital placé au fond d'un atrium génital de grandes dımensıons à 4 à $500 \mu$ de diamètre et de profondeur et dont la paroi musculeuse bien développée est, elle aussı, ornée de papilles.

C. popillotus (GRÉTILLAT, 1962)

13) Pore génital placé au fond d'un atrium génital dont la parol n'a pas de mus. culature bien définie.

14) Pore génital ne comportant pas d'atrium génital distinct. Papilles de très petites dimensions ( $10 \mu$ environ)..........

C. schoutedeni (GRÉTILLAT, 1964)

15) Papilles ornant le sphincter génital, l'atrıum génital ef débordant sur les parois de la poche ventrale; sphincter atrial bien développé ...........

.. C. endopapillatus (DOLLFUS, 1962)

16) Papilles ornant un atrium génital de dimensions appréciables .......... $\ldots \ldots \ldots$ C. wenyoni (LEIPER, 1908)

17) Papilles de très petite tailie 8 d̀ $10 \mu$, ornant le pore génital et un atrium génital très réduit, mais circonscrit par un sphincter très puissant .......

C. parvipapillatus, (GRÉTILLAT, 1962)

A l'examen de cette clé, nous voyons que les Carmyerius peuvent grossièrement se classer en trois groupes :

10 Le groupe synethes.

$2^{\circ}$ Le groupe graberı.

$3^{\circ}$ Le groupe gregarius.

$1^{0}$ Le groupe synethes qui comprend :

- C. synethes (FISCHOEDER, 1901).

- C. wenyoni (LEIPER, 1908).

- C. endopopillatus (DOLLFUS, 1962).

- C. papillatus (GRÉTILLAT, 1962).

L'atrium génital esł profond, bien développé ; l'absence ou la présence de papilles ainsi que l'épaisseur de la paroi de l'atrium et l'existence d'un sphincter sur le pourtour de l'orifice atrial permet de séparer ces quatre espèces.

20 Le groupe graberı qui comprend :

- C. graberı (GRÉTILLAT, 1960).

- C. exoporus (MAPLESTONE, 1923).

L'atrıum génital est d'importance réduite mais Il existe un très fort sphincter atrial. Chez $C$. exoporus, l'aire génitale est en dehors de la poche centrale alors que chez $C$. graber, elle se trouve à l'intérieur de cette poche.

30 Le groupe gregarius qui comprend :

- C. gregarus (LOOSS, 1896).

- C. spatiosus (BRANDES, 1898).

- C. welmoni (STYLES et GOLDBERGER, 1910).

- C. minutus (FISCHOEDER, 1901).

- $C$. dollfusi (GOLVAN, CHABAUD et GRÉTILLAT, 1957). 
- C. mancupatus (FISCHOEDER, 1901).

- C. porvipapillatus (GRÉTILLAT, 1962).

- C. schoutedeni (GRÉTILLAT, 1964).

II n'exıste pratiquement pas d'atrium génital: les seuls caractères permettant de différencier les espèces entre elles consistent dans des diffé- rences de structure' histologique et la présence ou l'absence de papilles.

Nous pensons cinisi que tout au moins pour dix espèces de Carmyerius, la diagnose peut être très facilement faite sur simples coupes sagittales, obtenues au rasoir à main, colorées ensuite au carmin chlorhydrique.
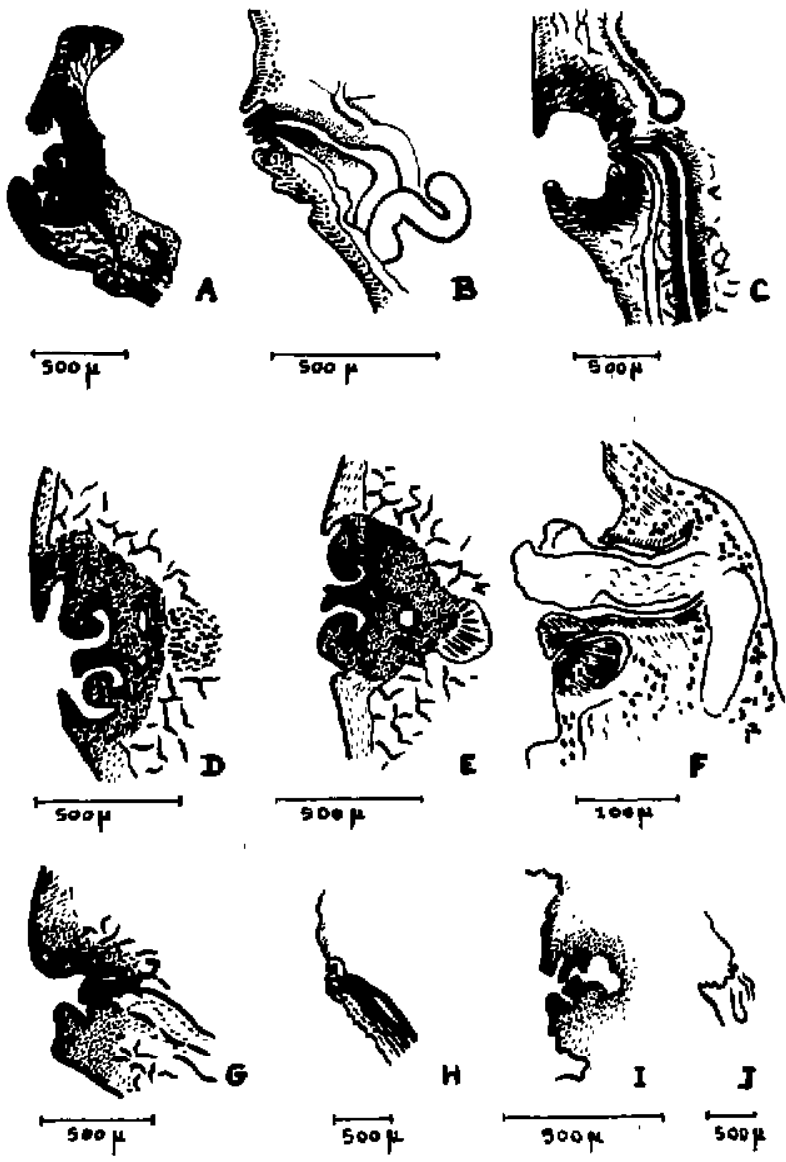

Fig. 1.

Pores génitaux chez:
A. C. graberı GRÉTILLAT, 1960.
B. C. minutus (FISCHOEDER, 1901) (d'après FISCHOEDER).
C. C. synethes (FISCHOEDER, 1901) (d'après FISCHOEDER).
D. C. spatiosus (BRANDES, 1898) (original).
E. C. gregarius (LOOSS, 1896) (orıgInal).
F. C. dollfist GOLVAN, CHABAUD et GRÉTILLAT, 1957.
G. C. exoporus MAPLESTONE, 1923 (d'après MAPLESTONE),
H. C. wellmani STILES et GOLD., 1910 (d'après STILES et GOLD.).
I, C. mancupatus (FISCHOEDER, 1901) (original).
J. C. wenyoni (LEIPER, 1900) (d'après WENYON). 
-

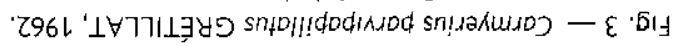

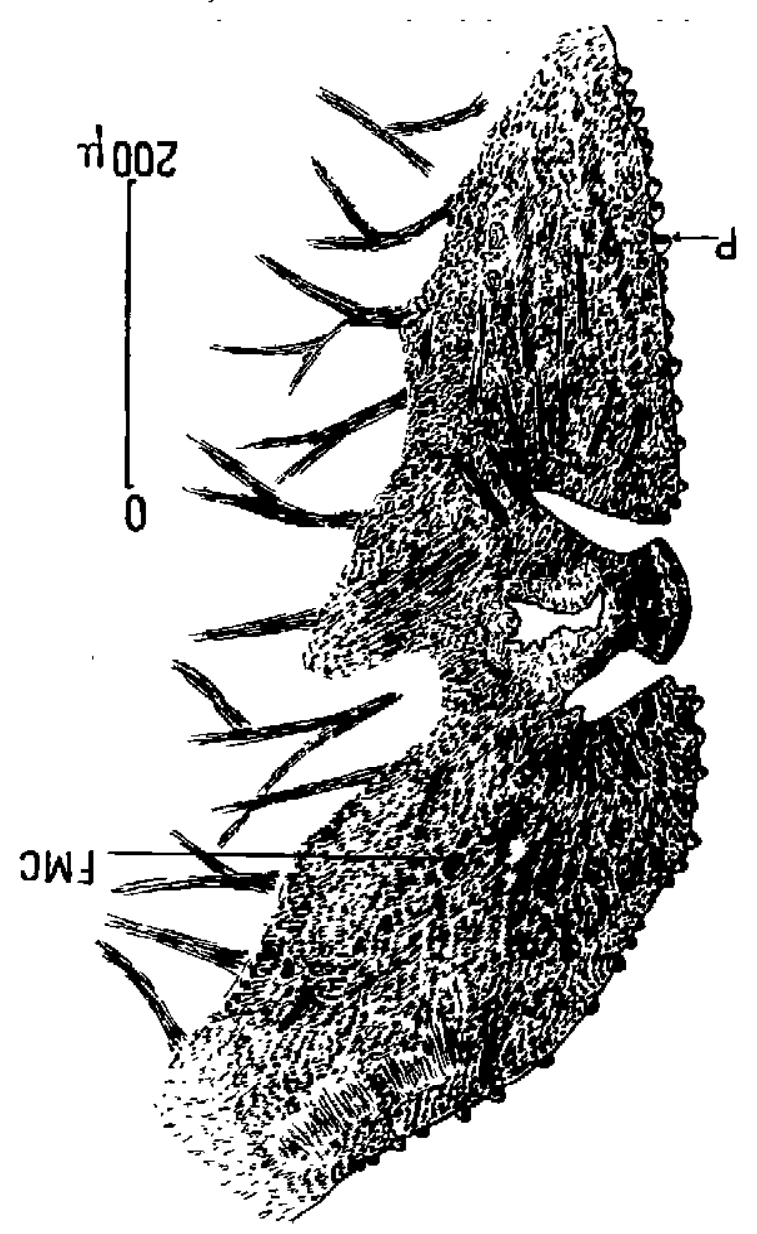

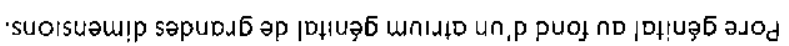

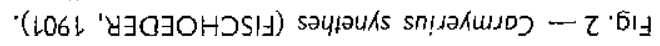

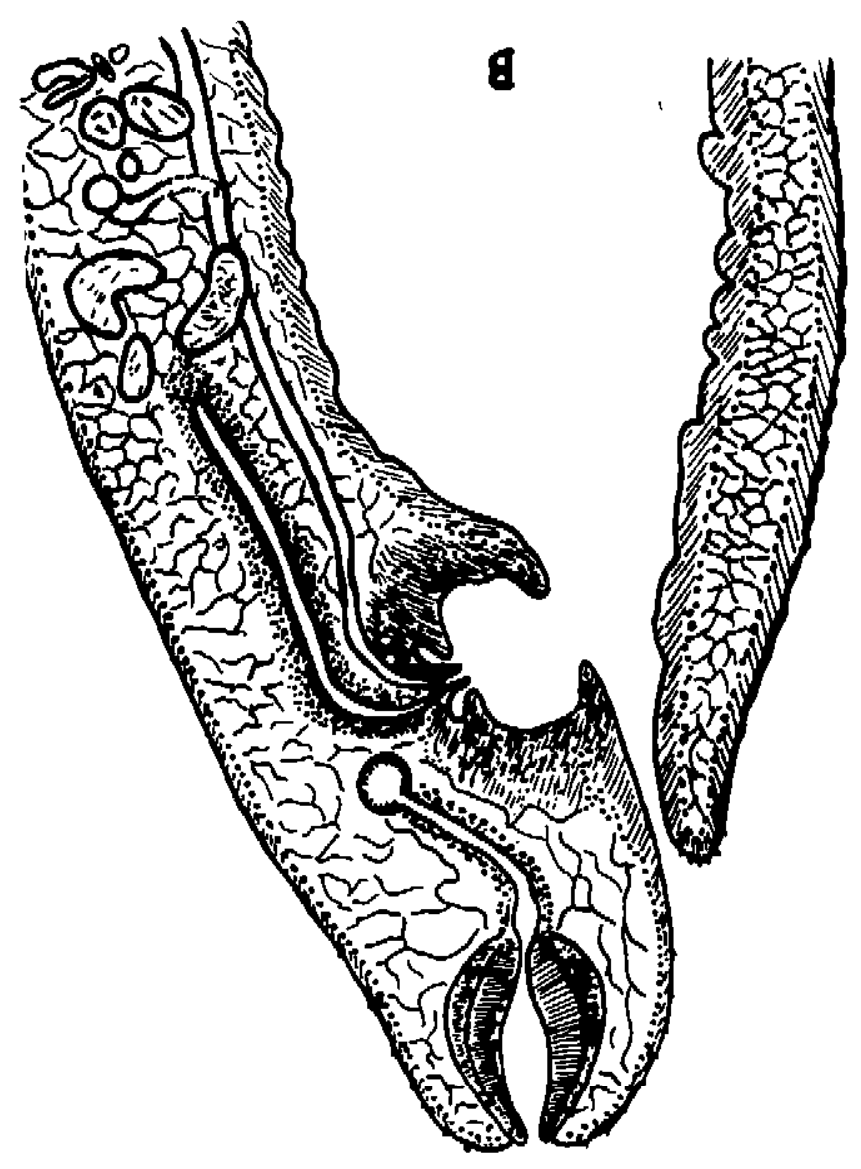




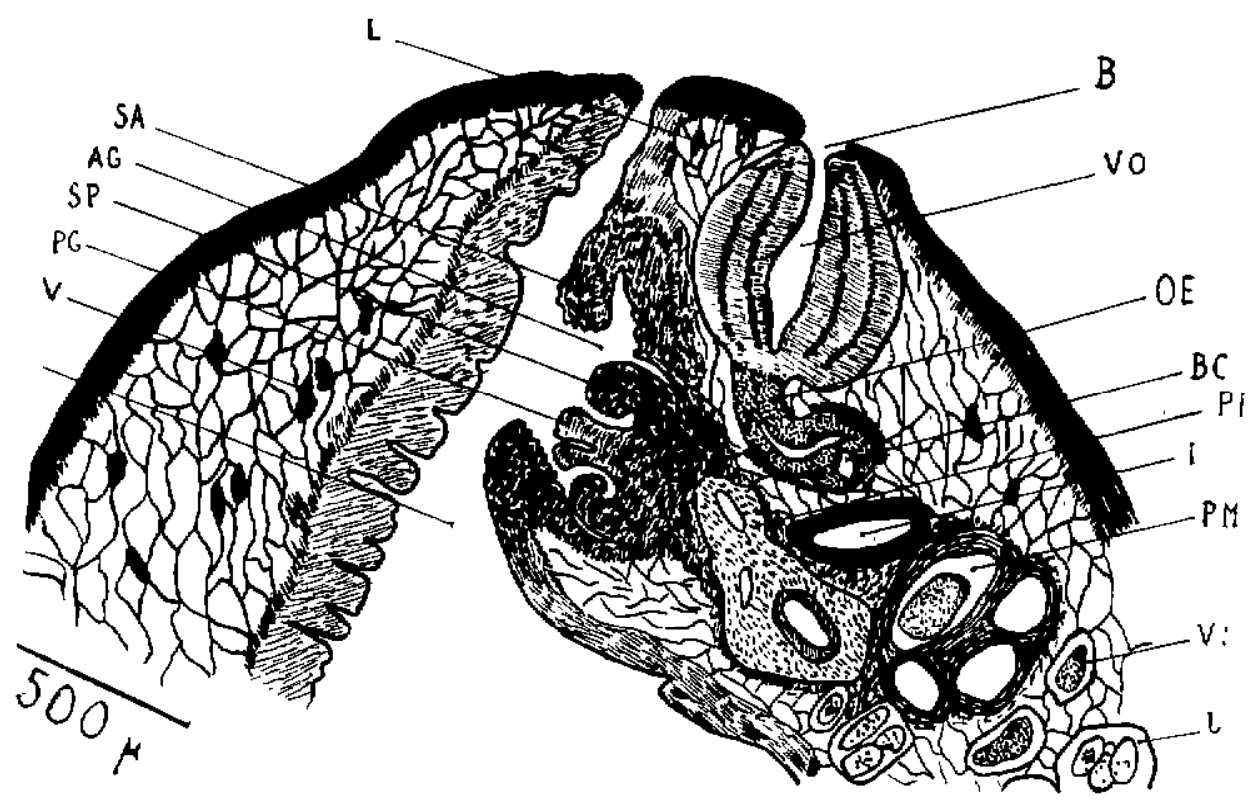

Fig. 4 - Cormyerus groberi GRÉTILLAT, 1960.

Pore génital.

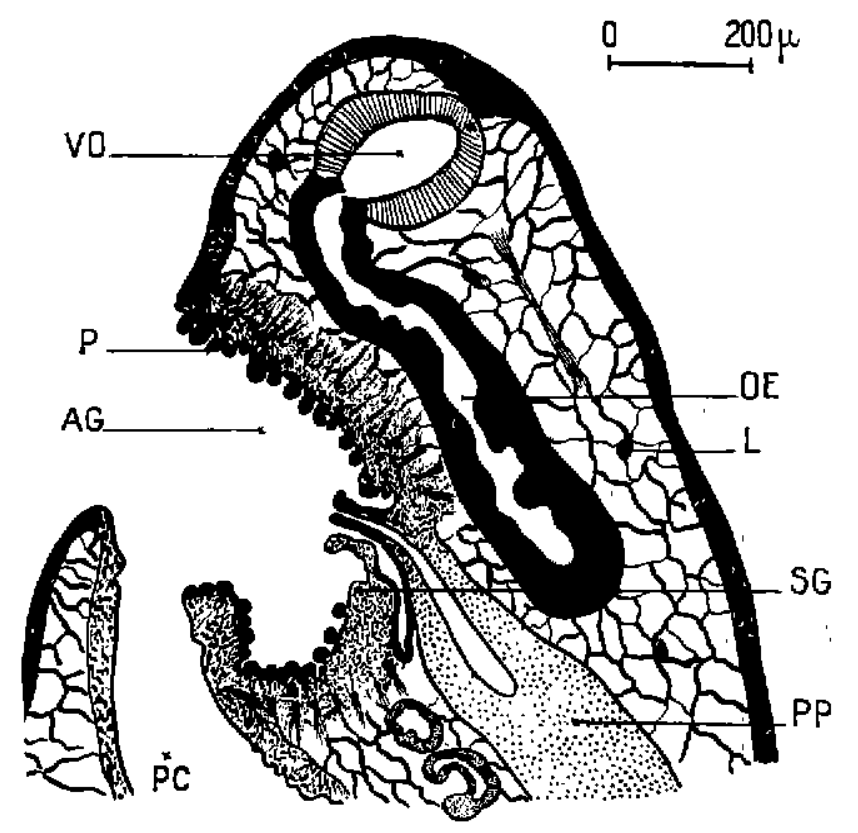

Fig. 5 - Cormyerius popillatus GRÉTILLAT, 1962.

Pore génital du fype synethes mais avec ałrıum génital à porois recouvertes de papilles. 


\section{SUMMARY}

The value of the morphological and anatomical characteristics of the genital pore in the classification af trematodes of the genus Cormyerius (Gastrathylocidae).

The classification of the Gostrothylacidae, particularly members of the genus Carmyerius (STILES and GOLDBERGER, 1910), is somewhat confused, especialty regarding those species where the morphological criteria are solely the size of the testicles, the diameter of the l'acetabulum, etc, characteristics which vary greatly when one consider how the contraction of preserved material can change greatly the size and aspect of certain organs in this group.

However, having studied the histological structure of the genital pore of the various members of this genus, it appears passible to classify some of them at least by taking as criteria the presence or absence, the aspect and size of :

10 the genital atruum,

$2^{\circ}$ the atrial sphincter,

$3^{\circ}$ the papillary sphincter.

By this means it has been possible to determine definitely eleven species out of the sixteen already described at the present time.

\section{RESUMEN}

Valor faxonómico de los caracteres morfológicos y anatómicos del poro genito en lo stremátodos del genero Carmyerius (Gosiroihylocidae).

La sistemâtica de los Gastrothylacidae, particularmente la de las especies del genero Cormyeruus STILES et GOLDBERGER, 1910, es más o menos confusa, por to menos en cuanto a ciertas especies se refiere en las cuales los criterios morfológicos, son dados solamente para las dimensiones de los testiculos, el diametro del acetábulo, etc..., caracteres muy fluctuantes si se considera que en este grupo la contracción del malerial conservado puede influir considerablemente en el aspecto y las dimensiones de ciertos órganos.

En cambio, después de haber estudıado la estrúctura histologica del poro genital de las diferentes especies del genero, parece ser posible clasificar estos ultimas (por lo menos algunas de ellas) tomando como criterios la existencia o la no-existencia, la imporlancía y las dimensiones :

10 del atrio genital.

$2^{\circ}$ del esfinter atrial,

30 del esfinter papilar.

Gracias a estos criterıos, es posible determinar con certitud ance especies entre las diez $y$ seis descritas ya actualmente.

\section{BIBLIOGRAPHIE}

BRANDES (G. Ph. H.). - Die Gattung Gastrothylax Abhandl. Naturforsh. Gesselsch. zu Halle (1898), Bd. XXI, pp. 195-225.

DOLLFUS (R. Ph.). - Variations intra-spécifiques chez un Carmyerius (Trematoda, Gastrothylacidae) parasite du buffle du Congo Belge. Ann. Porasit. Hum. Comp. (1962), t. XXXVII, no 1-2, pp. 108-120.
DOLLFUS ( $R$. Ph.). - Hôtes ef lieux de récolte de quelques trématodes digénétiques de Vertébrés de la Collection du Musée Royal de l'Afrique Centrale. Rev. Zool. Bot. africornes (1963), vol. LXVIII, fasc. 3-4, pp. 323-357.

FISCHOEDER (F.). - Die Paramphistomiden der Saügetiere Zoolog. Anzerger (1901) Bd. XXIV. no 646, pp. 367-375. 
FISCHOEDER (F.). - Die Paramphistomiden der Saügetiere Zoolog. Jahrbucher (1903) System, Bd XVII, Heft 4-6, pp. 485-660.

GOLVAN (Y. J.), CHABAUD (A. G.) et GRÉTILLAT (S.). - Carmyerius dollfusi n. sp. (Trematodo, Gostrothylacidae) parasite des bovidés de Madagascar Ann. Parasit. Hum. Comp. (1957), t. XXXI, no 1-2, pp. 56-70.

GRÉTILLAT (S.). - Amphıstomes des ruminants domestiques de la République du Tchad, description d'un Gostrothylacidae nouveau, Carmyerius graberi n. sp. Ann. Parasti. Hum. Comp. (1960), t. XXXV, no 4, pp. 509-527.

GRÉTILLAT (S.). - Carmyerius papillatus n. sp. et Carmyerius parvipapillofus n. sp. (Trematoda, Gastrothylacidae). parasites des réservoirs gastriques de l'antilope Kobus defassa Rüpp. Ann. Parasit. Hum. Comp., t. XXXVII, (1962), no 1-2, pp. 121-139.

GRÉTILLAT (S.). - Sur quelques Paramphistomatoidea (Trematoda) d'une collection du Musée Royal de l'Afrique Centrale. Revue de Zaologie ef de Botanique Africanes, en cours de publications (1964).

INNES (J. I.). - Gastrothylax bubalis n. sp., with a few notes on the genus Gastrothylax. (Poirier) Parasitology (1912), vol. V, no 3 , PP. 217-222.

TLEPER (R.T.). - The entozod of the Hippopotomus. Proc. Zool. Soc, London (1908) n० 1 , P. 233-251.

LOOSS (A.). - Recherches sur la faune parasitaire de l'Egypte. Première partie. Mem. Inst. d'Egypte (1896), Le Caire, t. III, pp. 1-252.

MAPLESTONE (P. A.). - A revision of the Amphistomata of Mammals. Ann. Trop. Med. Parasit. (1923), vol: XVII, no 2, PP. 113-212.

STILES (Ch. W.) and GOLDBERGER (J.). - A study of the anatomy of Watsonius n. $g$. watsoni of man and nineteen allied species of mammalian Trematode worms of the superfamily Paramphistomatoidea. Hyg. Labor. Bull. (1910), no 60, 259 pages, April 1910, Washington. 